\title{
Targeting Seizure-Induced Neurogenesis in a Clinically Relevant Time Period Leads to Transient But Not Persistent Seizure Reduction
}

\author{
Parul Varma, ${ }^{1}$ Rebecca Brulet, ${ }^{2}$ Ling Zhang, ${ }^{2}$ and Jenny Hsieh ${ }^{1}$ \\ ${ }^{1}$ Department of Biology and Brain Health Consortium, University of Texas at San Antonio, San Antonio, Texas 78249, and ${ }^{2}$ Department of Molecular \\ Biology, University of Texas Southwestern Medical Center, Dallas, Texas 75390
}

\begin{abstract}
Mesial temporal lobe epilepsy (mTLE), the most common form of medically refractory epilepsy in adults, is usually associated with hippocampal pathophysiology. Using rodent models of mTLE, many studies including work from our laboratory have shown that new neurons born around the onset of severe acute seizures known as status epilepticus (SE) are crucial for the process of epileptogenesis and targeting seizure-induced neurogenesis either genetically or pharmacologically can impact the frequency of chronic seizures. However, these studies are limited in their clinical relevance as none of them determines the potential of blocking new neurons generated after the epileptogenic insult to alleviate the development of chronic seizures. Therefore, using a pilocarpine-induced SE model of mTLE in mice of either sex, we show that $>4$ weeks of continuous and concurrent ablation of seizure-induced neurogenesis after SE can reduce the formation of spontaneous recurrent seizures by $65 \%$. We also found that blocking post-SE neurogenesis does not lead to long-term seizure reduction as the effect was observed only transiently for $10 \mathrm{~d}$ with $>4$ weeks of continuous and concurrent ablation of seizureinduced neurogenesis. Thus, these findings provide evidence that seizure-induced neurogenesis when adequately reduced in a clinically relevant time period has the potential to transiently suppress recurrent seizures, but additional mechanisms need to be targeted to permanently prevent epilepsy development.
\end{abstract}

Key words: adult neurogenesis; epilepsy; hippocampus; mTLE; neural stem cell; newborn neurons

Significance Statement

Consistent with morphological and electrophysiological studies suggesting aberrant adult-generated neurons contribute to epilepsy development, ablation of seizure-induced new neurons at the time of the initial insult reduces the frequency of recurrent seizures. In this study, we show that continuous targeting of post-insult new neurons in a therapeutically relevant time period reduces chronic seizures; however, this effect does not persist suggesting possible additional mechanisms.

\section{Introduction}

Millions of people around the world suffer from epilepsy, a neurological disorder where highly synchronized and highfrequency activity of neurons leads to epileptic discharges. Mesial

Received April 23, 2019; revised June 18, 2019; accepted June 20, 2019.

Author contributions: P.V. and J.H. designed research; P.V., R.B., and L.Z. performed research; P.V. and J.H. analyzed data; P.V. and J.H. wrote the paper.

This work was supported by Grants from the National Institute of Health (R01NS093992, R01NS081203, R01NS089770, and R21NS090926 to J.H.), Department of Defense (W81XWH-15-1-0399 to J.H.), American Heart Association (Grant 15GRNT25750034), by a postdoctoral fellowship from the American Epilepsy Society (Award 565714 to P.V.), and by the Robert J. Kleberg Jr. and Helen C. Kleberg Foundation and the Semmes Foundation. We thank Zane R Lybrand for providing comments on the paper, Jingfei Zhu for technical assistance, Jose Cabrera for help with the graphics, and Mary-Helen Mays for editing assistance.

The authors declare no competing financial interests.

Correspondence should be addressed to Jenny Hsieh at jenny.hsieh@utsa.edu.

https://doi.org/10.1523/JNEUROSCI.0920-19.2019

Copyright $\odot 2019$ the authors temporal lobe epilepsy (mTLE) is the most common form of medically refractory epilepsy in adults (Engel, 2001). Apart from genetic mutations and developmental malformations, brain injuries such as head trauma, infection, ischemia, prolonged febrile seizures and status epilepticus (SE) cause mTLE (Kwan and Brodie, 2000; Chang and Lowenstein, 2003). Furthermore, such an initial brain insult is followed by a latent period before clinically detectable seizures appear eventually culminating in spontaneous recurrent seizures (SRS). This latent period is also described as the period of epileptogenesis and may present a possible therapeutic window to prevent the development of epilepsy (Pitkänen and Sutula, 2002).

Hippocampal sclerosis is the most common pathophysiology associated with mTLE but other structural lesions could also play a role in seizure development (Mathern et al., 1993, 1995; Semah et al., 1998). Although recent work has sparked debate regarding 
the level of adult hippocampal neurogenesis in humans, new neurons are continuously generated in the dentate gyrus of rodent hippocampus and shown to play essential roles in memory and cognition under physiological conditions (Kempermann et al., 2004, 2018; Gage and Temple, 2013; Epp et al., 2016; Boldrini et al., 2018; Sorrells et al., 2018; Gage, 2019; Moreno-Jiménez et al., 2019). Moreover, abnormal granule cells have been observed in both rodent models of mTLE and human mTLE patients although the contribution of new neurons to this process is not known (Parent et al., 1997; von Campe et al., 1997; Marucci et al., 2013). mTLE is characterized by abnormal maturation or morphology in hippocampal neurogenesis including production of ectopic granule cells (EGCs), mossy fiber sprouting, neuronal hypertrophy, persistence of hilar basal dendrites, and accelerated maturation (Parent et al., 1997; Nadler, 2003; OverstreetWadiche et al., 2006; Thind et al., 2008). These morphological and functional abnormalities in adult-generated neurons are thought to contribute to the development of epileptogenic circuitry (Scharfman, 2002; Scharfman et al., 2007). Studies using rodent models of mTLE have shown that by reducing adult hippocampal neurogenesis either genetically or pharmacologically, the frequency of chronic seizures is altered suggesting that seizure-induced neurogenesis represents an important cellular target to prevent epilepsy (Jung et al., 2004, 2006; Cho et al., 2015; Hosford et al., 2016, 2017).

However, a limitation of these studies is that ablation of neurogenesis happens before the acute seizures, which has limited therapeutic relevance. For example, Cho et al. (2015) showed that blocking neurogenesis for 4 weeks before pilocarpine-induced SE led to seizure suppression. Consistent with these findings, Hosford et al. (2016)showed a reduction in SRS when the cohort of cells born up to 5 weeks before pilocarpine-induced SE was labeled and ablated beginning $3 \mathrm{~d}$ after the induction of SE. Interestingly, Hosford et al. (2017) performed a subsequent study in which cells born before and after SE were labeled but ablated 2-3 months after the animals were in the chronic seizure phase, halted epilepsy development but did not cause an immediate reduction in the seizures. Though all of these studies support the notion that new neurons born around the time of the initial insult contribute to epileptogenesis, whether it is possible to exclusively target post-SE generated neurons to reduce recurrent seizures is still unknown.

Based on these previous studies, we hypothesized that ablation of post-seizure neurogenesis would suppress epilepsy formation. To test our hypothesis, we used Nestin- $\delta$-HSV-thymidine kinase-EGFP (Nestin-TK) transgenic mice to ablate neurogenesis for 4 or 8 weeks specifically after SE in a mouse pilocarpineinduced SE model of mTLE. We measured SRS frequency from 5 weeks post-SE (WPSE) to 7-WPSE and found that $>4$ weeks of continuous ablation of post-SE neurogenesis was required to reduce SRS development by $65 \%$. We also found that this reduction in SRS was transient for $10 \mathrm{~d}$ with $>4$ weeks of continuous and concurrent ablation of seizure-induced neurogenesis. Thus, we show that continuous ablation of seizure-induced neurogenesis has the potential to control the development of chronic seizures at least transiently.

\section{Materials and Methods}

Mice. Both male and female mice were used in this study. Mice were bred and maintained as per the guidelines of the animal facility including $12 \mathrm{~h}$ light/dark cycles and not $>5$ animals per cage. Nestin-TK-EGFP mice were bred and genotyped as described previously (Cho et al., 2015). Alzet mini osmotic pumps (model 2004) were used in all the experiments for the delivery of Vehicle (saline) or ganciclovir (GCV; PRX315110) to the animals. The GCV dose used for the experiments was $150 \mathrm{mg} / \mathrm{kg} / \mathrm{d}$ and the pumps were replaced after 4 weeks in the experiments where two rounds of pumps were used. All the experiments were performed in compliance with the regulatory guidelines for animal care issued by the National Institutes of Health and by the Institutional Animal Use and Care Committee at University of Texas Southwestern Medical Center, Dallas, TX.

Chemoconvulsant model of mTLE. Six-week-old male and female Nestin-TK mice were administered scopolamine methyl nitrate $(2 \mathrm{mg} /$ $\mathrm{kg}$; Sigma-Aldrich, S2250) and terbutaline hemisulfate salt $(2 \mathrm{mg} / \mathrm{kg}$; Sigma-Aldrich, T2528) intraperitoneally to block peripheral effects of pilocarpine and dilate the respiratory tract, respectively. Thirty minutes later, mice were intraperitoneally injected with pilocarpine hydrochloride (Sigma-Aldrich, P6503) at $245 \mathrm{mg} / \mathrm{kg}$ for males and $280 \mathrm{mg} / \mathrm{kg}$ for females and placed in an incubator (Thermocare) maintained at $31^{\circ} \mathrm{C}$. Acute seizures were behaviorally monitored based on a modified Racine scale (Racine, 1972) with different stages as following: Stage 1, mouth and facial movement; Stage 2, head nodding; Stage 3, forelimb clonus; Stage 4 , rearing with forelimb clonus; Stage 5, rearing and falling with forelimb clonus. Once the mice reached SE (defined by continuous tonic clonic convulsive seizures), they were placed at room temperature (RT) for $3 \mathrm{~h}$. Diazepam (Sigma-Aldrich, D0899) was administered intraperitoneally at $10 \mathrm{mg} / \mathrm{kg}$ to mice after $3 \mathrm{~h}$ to stop the seizures and mini osmotic pumps with either Veh or GCV were implanted subcutaneously. To facilitate the recovery process, mice were administered $5 \%$ dextrose solution $(1 \mathrm{ml}$, i.p.) and $0.9 \%$ saline $(1 \mathrm{ml}$, i.p.). Mice were monitored in the incubated chamber for $2 \mathrm{~d}$ after which they were returned to home cages and housed together. All animals used in the study were injected with bromodeoxyuridine (BrdU; 150 mg/kg, i.p.; Sigma-Aldrich B5002) once a day on Days 1-3 after pilocarpine to label proliferating cells after acute seizures.

Video-electroencephalogram monitoring. Video-electroencephalogram (EEG) recording was performed between 5-WPSE and 7-WPSE and in other cases 18-WPSE to 20-WPSE. Mice were anesthetized using 2-3\% isoflurane and were given analgesic buprenorphine $(0.05 \mathrm{mg} / \mathrm{kg}$, s.c.) before the surgery as per animal care guidelines. EEG transmitters (TA11ETAF10, Data Sciences International) were implanted subcutaneously with wires connected to stainless steel screws drilled in the skull at the following coordinates from bregma: Screw 1 at anterior-posterior (AP): +0.1 , medial lateral (ML): +0.2 ; and Screw 2 at AP: -0.2 , ML: +0.22 . Continuous video-EEG monitoring via wireless telemetry was performed in singly housed mice for a total of 2 weeks. The data were reviewed and quantified by a user blinded to the experimental groups using NeuroScore Software v3.0 (Data Sciences International). Behavioral seizures were defined by repetitive epileptiform spiking activity $(\geq 3 \mathrm{~Hz}$ ) that persisted for $>10 \mathrm{~s}$ and was confirmed using video recordings (Cho et al., 2015). Convulsive seizure activity was marked at the beginning and end of each event to account for seizure duration and the number of seizures for each mouse. In our analysis, all mice with EEG seizures also showed convulsive seizures and we were not able to define any non-convulsive seizures (Kelly, 2004; D’Ambrosio and Miller, 2010; Dudek and Bertram, 2010). Therefore, to avoid any ambiguity in data analysis, we measured the convulsive seizures that were robust, and we could confirm them with the corresponding videos based on the Racine scale. The time course analysis of seizure development over $14 \mathrm{~d}$ was performed by dividing the entire time period into 5 epochs $(0-2,2-5,5-8,8-11,11-14 \mathrm{~d})$ and the average number of seizure events per epoch were calculated for both Veh- and GCV-treated animals. At the end of EEG recordings, mice were killed and brains collected for immunohistochemistry.

Immunohistochemistry. All the mice after EEG recordings were anesthetized and transcardially perfused with $4 \%$ cold paraformaldehyde (PFA; Fisher Scientific, AC416780100). The brains were dissected out and transferred to $4 \%$ PFA for $1 \mathrm{~d}$ following which they were transferred to $30 \%$ sucrose solution where they were allowed to sink to the bottom of the tubes until $2 \mathrm{~d}$. The fixed brains were bisected and right side of the brain was coronally sectioned at $30 \mu \mathrm{m}$ thickness on a freezing microtome (Leica, SM 2000R). All doublecortin (DCX) and Prospero ho- 
meobox (Prox1) staining were performed on free-floating tissue sections as detailed in the following protocol. Free floating tissue sections were washed thrice in $1 \times$ Tris-buffered saline (TBS) and treated with 3\% $\mathrm{H}_{2} \mathrm{O}_{2}$ (Fisher Scientific, H325) for $30 \mathrm{~min}$ at RT on a shaker to quench the endogenous peroxidases. After $30 \mathrm{~min}$, the sections were thoroughly rinsed with $1 \times$ TBS 3-4 times and were left in blocking solution made of $3 \%$ donkey serum (Sigma-Aldrich, D9663) and 0.3\% Triton X-100 (Fisher Scientific, BP151) in $1 \times$ TBS for $1 \mathrm{~h}$ at RT. Goat anti-DCX (1:1000; Santa Cruz Biotechnology, sc-271390) or rabbit anti-Prox1 (1: 1000; Millipore, AB5475) was added and the sections were left overnight at $4^{\circ} \mathrm{C}$. On Day 2 , the sections were washed with $1 \times$ TBS $3-4$ times, incubated in goat anti-biotin (1:200; Jackson ImmunoResearch, 705065-147) or rabbit anti-biotin (1:20; Jackson ImmunoResearch, 711065-152) for $2 \mathrm{~h}$ at RT. The sections were subsequently washed 3-4 times with $1 \times$ TBS and incubated in avidin biotin complex (1:50; ABC Vectastain, Vector Laboratories, PK-4000) in $1 \times$ TBS for $1 \mathrm{~h}$ at RT. After ABC treatment, sections were rinsed 3-4 times in $1 \times \mathrm{TBS}$, treated with 3 , 3'-diaminobenzidine (DAB + chromogen; Dako, K3468) for 2-3 min, rinsed thoroughly with $1 \times$ TBS and mounted on microscopic slides. The slides were air-dried and serially dehydrated with 70, 80, 90, 95, and $100 \%$ ethyl alcohol for $3 \mathrm{~min}$ each. After $100 \%$ alcohol, the slides were treated with xylene for $30 \mathrm{~min}$, cleaned and coverslipped. For BrdU/ Proxl staining, sections were washed twice with $1 \times$ TBS and permeabilized with $0.4 \%$ Triton in TBS for $30 \mathrm{~min}$ at RT. After permeabilization, the sections were washed twice with $1 \times$ TBS for 10 min each, following which they were incubated in $2 \mathrm{~N}$ hydrochloric acid $(\mathrm{HCl})$ at $37^{\circ} \mathrm{C}$ for 15 min. After $\mathrm{HCl}$ treatment, the sections were washed with $0.1 \mathrm{M} \mathrm{Na}_{2} \mathrm{~B}_{4} \mathrm{O}_{7}$ (Borate buffer), pH 9.5, for $10 \mathrm{~min}$ at RT. They were subsequently washed twice with $1 \times$ TBS at RT and incubated in blocking buffer for $1 \mathrm{~h}$. Rat anti-BrdU (1:500; Abcam, ab6326) and rabbit anti-Proxl (1:500; Millipore, AB5475) were added onto the sections and left overnight at $4^{\circ} \mathrm{C}$. On Day 2, the sections were rinsed 3-4 times in $1 \times$ TBS and incubated with secondary antibodies (1:300, anti-rat-cy3; Jackson ImmunoResearch, 712-165-153; and 1:300, anti-rabbit AlexaFluor 488, Al-11055) for $3 \mathrm{~h}$. The sections were then rinsed thrice in $1 \times$ TBS for 10 min each at RT, DAPI was added and rinsed again and mounted on the microscopic glass slides.

Microscopic analysis and quantification. Quantification of cell number for the DAB stained cells in the hippocampus was performed using an inverted microscope (Nikon, Eclipse TE2000-U) equipped with a colored camera by an observer blinded to experimental groups. Subgranular zone was defined as the area within the diameter of one granule cell from the margin of granule cell layer. For counting hilar ectopic granule cells, hilar zone was defined as the area beyond the granule cell layer. Immunoreactive cells were quantified in every twelfth $30 \mu \mathrm{m}$ coronal section throughout the dentate gyrus. The numbers counted from each section were added and multiplied by 24 to estimate the total number of cells in one animal brain. Leica SP8 was used for confocal imaging and ImageJ was used for image analysis.

Statistics. All the data are expressed as mean \pm SEM. Experimental groups were assigned by simple randomization. No statistical methods were used to predetermine sample sizes. Statistical differences were analyzed using two-tailed Student's $t$ test for data with equal variances. None of the results showed statistically significant sexual dimorphism. Oneway ANOVA was used for comparison between data with multiple groups. GraphPad Prism software v7.0 was used for all statistical analysis. Values of $p<0.05$ were considered significant.

\section{Results}

\section{Four weeks of blocking post-seizure neurogenesis does not suppress chronic seizures}

To determine whether 4 weeks of blocking post-seizure neurogenesis can suppress chronic seizures, we used Nestin-TK transgenic mice to ablate adult hippocampal neurogenesis after acute seizures (Fig. 1A). With this genetic model, dividing neural progenitor cells can be specifically ablated with the administration of GCV without affecting glial and endothelial cells (Yu et al., 2008). Approximately 6-week-old male and female mice were injected with pilocarpine to induce SE and allowed to remain in SE for $3 \mathrm{~h}$ before terminating the seizures with diazepam. Immediately after diazepam injections, osmotic minipumps filled with either GCV or Veh were implanted in the mice to ablate neurogenesis for 4 weeks. To assess the functional impact of blocking post-seizure neurogenesis, we performed 2 weeks (5-WPSE to 7-WPSE) of 24/7 continuous video-EEG monitoring. We found that 4 weeks of ablation did not have any effect on SRS frequency or duration in GCV-treated animals compared with Veh-treated controls (Fig. 1 B, C) suggesting that this amount of ablation is not enough to reduce seizure-induced new neurons to affect SRS. Though there was no overall significant difference in SRS between Vehand GCV-treated animals, we found that SRS frequency evolved over the period of $14 \mathrm{~d}$ where Veh-treated animals had more seizures compared with GCV-treated animals. This suggested that without treatment control animals might continue to have more seizures and continuous ablation might help in effectively reducing the SRS frequency (Fig. 1D). It has been shown that seizures induce proliferation of DCX-expressing late stage progenitors and neuroblasts in the subgranular zone (SGZ) of DG (Jessberger et al., 2005). Additionally, seizures also increase the number of granule cells located ectopically in the dentate hilus (Scharfman et al., 2007). Therefore, to gain insight into the cellular changes affected by 4 weeks of post-seizure ablation, we first assessed the number of DCX-expressing neuroblasts and immature neurons in the SGZ. We found that whereas Veh-treated animals had 10,581 $\pm 921.2(n=7)$ DCX + cells, GCV-treated animals showed a significant reduction to $1450 \pm 255.2(n=7)$ cells in the SGZ (Fig. 1E,F). Next, we examined the Proxl expressing hilar EGCs in the dentate hilus. Whereas Veh-treated animals had $7615 \pm 948.6(n=7)$ hilar EGCs, the number of hilar EGCs in GCV-treated animals was significantly decreased to $1903 \pm 318.2(n=7$; Fig. $1 G, H)$. Thus, despite the significant reduction in post-seizure neurogenesis, we found that SRS persist, suggesting that seizure-induced neurogenesis needs to be continuously reduced or reduced below a certain threshold to impact SRS frequency (Brulet et al., 2017).

\section{Greater than 4 weeks of blocking post-seizure neurogenesis suppresses chronic seizures}

To test the hypothesis that seizure-induced neurogenesis needs to be continuously reduced to impact chronic SRS and because 4 weeks of ablation of post-seizure neurogenesis did not affect overall SRS development, we ablated neurogenesis for 8 weeks after SE (Fig. 2A). Osmotic minipumps filled with either Veh or GCV were inserted in Nestin-TK mice immediately after termination of 3 h of pilocarpine-induced SE. However, after 4 weeks of ablation, the first set of minipumps were removed, and a new set of pumps filled with Veh or GCV were inserted into these mice for 4 more weeks. We performed continuous video EEG monitoring for 2 weeks similar to the first experimental paradigm (5-WPSE to 7-WPSE) and found a 65\% reduction in SRS frequency in the GCV-treated group of animals with no change in SRS duration compared with Veh-treated animals (Fig. 2B,C). We also found that similar to the first experimental paradigm, the control animals continued to have more seizures, whereas the SRS frequency in GCV-treated animals dropped significantly. Interestingly, the significant seizure reduction was only in the first $10 \mathrm{~d}$ and started disappearing by the end of 2 weeks (Fig. 2D). Therefore, we did not continue to record for 1 more week while GCV was still given and perfused the mice at 8 weeks (after the completion of GCV treatment) and performed histology to estimate the reduction in DCX + neuroblasts and immature neurons 


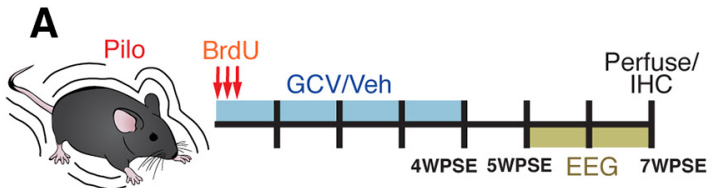

6 week old Nestin-TK
B

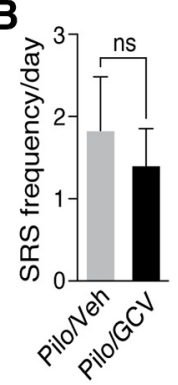

C

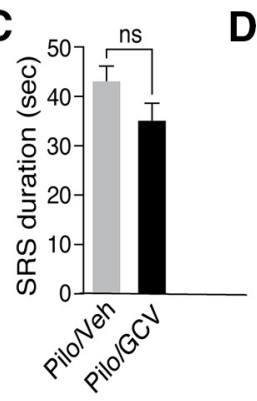

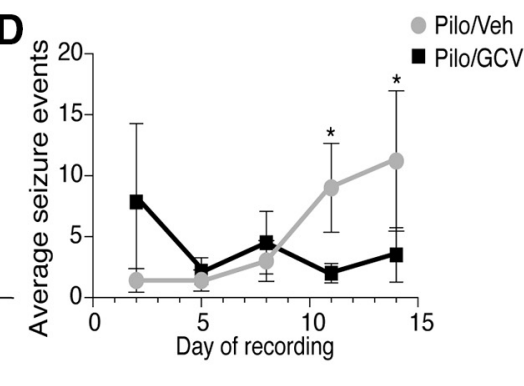

E

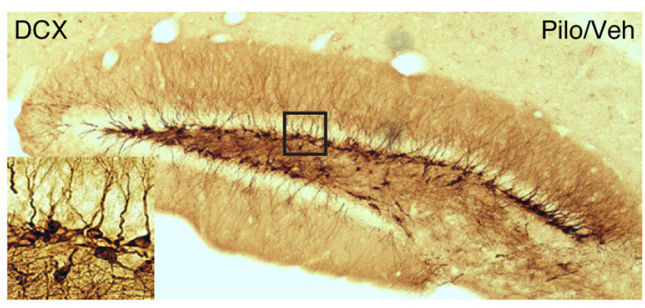

G

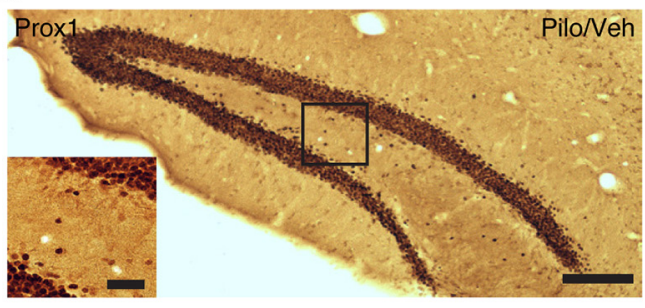

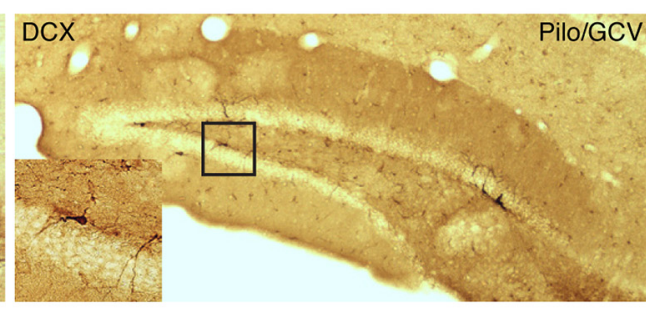

$F$

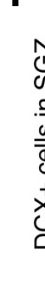

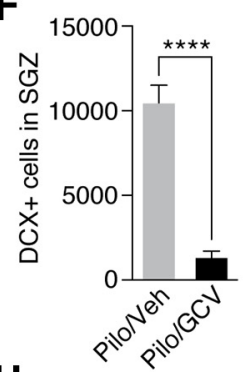

H
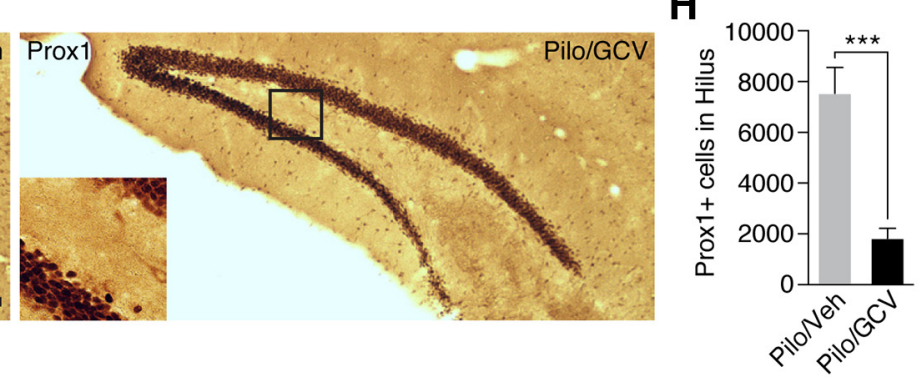

Figure 1. Four weeks of blocking post-seizure neurogenesis does not suppress chronic seizures. $A$, Experimental design. $B$, Graph showing frequency of SRS between Pilo/Veh and Pilo/GCV groups. Student's t test, $p=0.5772$. C, Graph showing SRS duration between Pilo/Veh and Pilo/GCV groups. Student's t test, $p=0.0808$. $D$, Graph showing time course analysis of seizure frequency of Pilo/Veh and Pilo/GCV groups over the period of $14 \mathrm{~d}$. $\boldsymbol{E}$, Representative microscopic images showing DCX immunoreactivity between Pilo/Veh and Pilo/GCV groups. Inset, Typical DCX+ cell. $\boldsymbol{F}$, Graph showing the number of DCX-expressing cells in the SGZ between the Pilo/Veh and Pilo/GCV groups. Student's $t$ test, $p<0.0001$. G, Representative microscopic images showing Prox 1 immunoreactivity of the mature granule neuron between Pilo/Veh and Pilo/GCV groups. Inset, Typical Prox1+ EGCs. H, Graph showing Prox1+ EGCs in the Pilo/Veh and Pilo/GCV groups. Student's $t$ test, $p<0.0001$. Scale bar, $100 \mu \mathrm{m}$; inset, $25 \mu \mathrm{m} . N=6-7$ animals per group. ns $=$ not significant. pilo, Pilocarpine. ${ }^{*} p<0.05,{ }^{* * *} p \leq 0.001,{ }^{* * * *} p \leq 0.0001$.

in SGZ and Prox1+ hilar EGCs. We found that with 8 weeks of post-seizure ablation of new neurons, there were only $656 \pm$ $379.2(n=9)$ DCX + cells in the SGZ of GCV-treated animals compared with $5892 \pm 1589(n=9)$ cells in Veh-treated animals (Fig. 2E,F). Likewise, we found a significant reduction in Prox $1+$ cells with $681 \pm 174.6(n=9)$ hilar EGCs in GCVtreated animals compared with the $4092 \pm 681.9(n=9)$ hilar EGCs in Veh-treated animals (Fig. 2G,H). Hence, continuous ablation of post-seizure neurogenesis for 8 weeks further reduced the seizure-induced new neurons compared with 4 weeks of ablation. This suggested that $>4$ weeks of continuous ablation of seizure-induced new neurons can reduce the development of chronic seizures.

\section{Reduced seizures from 8 weeks of post-seizure ablation does not persist}

Because $>4$ weeks of continuous and concurrent ablation of seizure-induced neurogenesis showed 65\% reduction in SRS for the first $10 \mathrm{~d}$ and started disappearing toward the end of 2 weeks, we decided to corroborate this finding by asking whether this loss in reduction of SRS at the end of 2 weeks is transient or persists even at later stages. To test this, we again ablated neurogenesis for 8 weeks after SE (Fig. 3A). However, after 8 weeks of ablation, we waited for 10 weeks before performing continuous video EEG monitoring for 2 weeks (18-WPSE to 20-WPSE). We found no change in SRS frequency and SRS duration between the Veh- and GCV-treated groups of animals (Fig. $3 B-D$ ). To provide a possible explanation why 8 weeks of post-seizure ablation led to shortbut not long-term SRS reduction, we performed histological analysis for DCX and Proxl. We found $3291 \pm 930(n=7)$ DCX + cells in the SGZ of Veh-treated group of animals compared with $921 \pm 321(n=5)$ in GCV-treated animals (Fig. $3 E, F)$. Furthermore, we found $8848 \pm 1127(n=7)$ Proxl+ hilar EGCs in Veh-treated animals compared with $1594 \pm 289.1$ $(n=5)$ hilar EGCs in GCV-treated animals (Fig. 3G,H). Thus, there was a greater reduction in seizure-induced new neurons at 8 -WPSE $(\sim 89 \%$ decrease in DCX + cells, $\sim 83 \%$ decrease in Prox $1+$ cells $)$ compared with 20 -WPSE $(\sim 72 \%$ decrease in DCX + cells, $\sim 81 \%$ decrease in Prox $1+$ cells) reinforcing the concept that post-SE neurogenesis has to be reduced continuously to impact the recurrent seizures.

\section{Effect of ablation of seizure-induced neurogenesis on survival of new neurons}

Seizures also increase the number of Prox $1+$ granule cells located ectopically in the dentate hilus (Scharfman et al., 2007). To determine how many neural progenitors at the time of acute seizures become hilar ectopic mature neurons, we examined the 

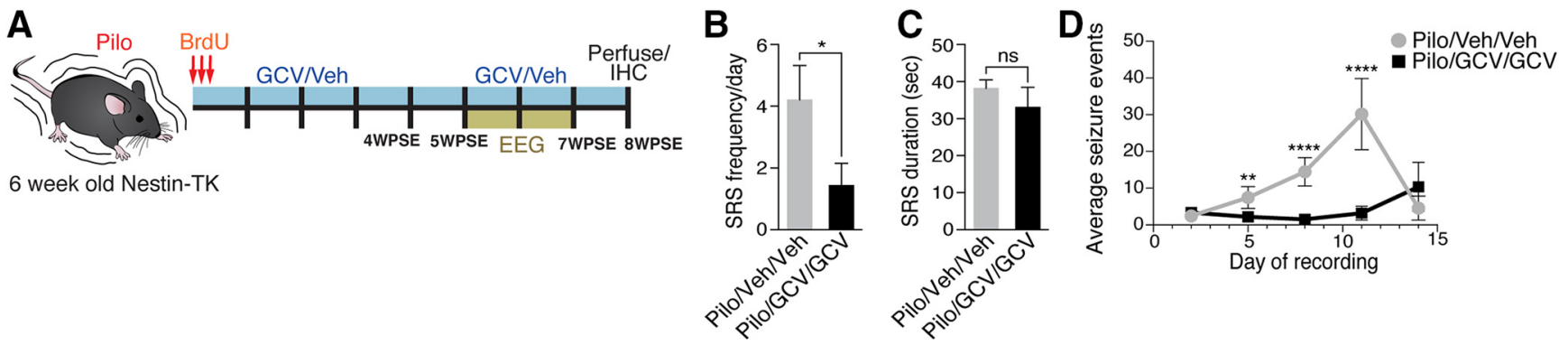

E
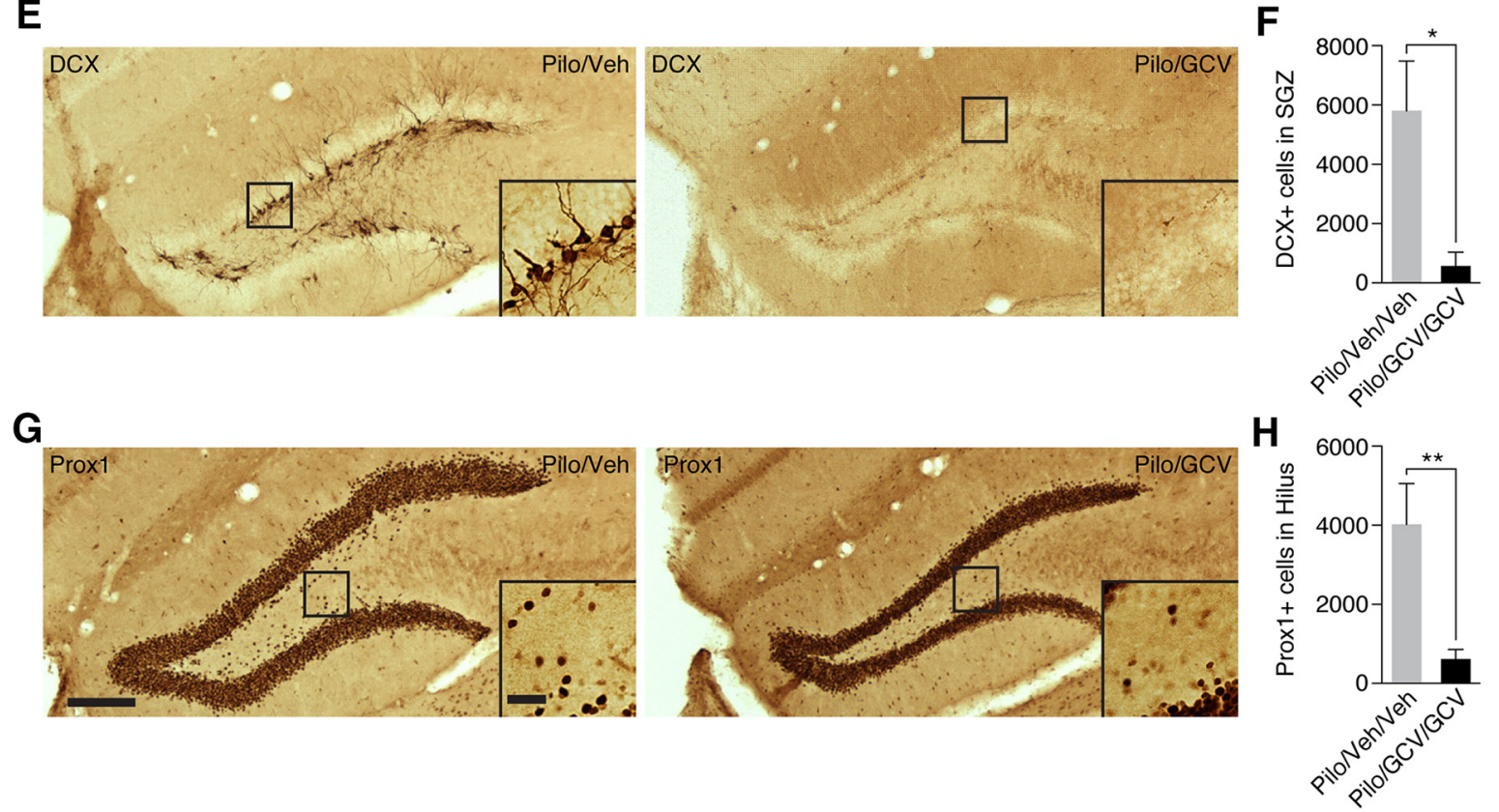

Figure 2. Greater than 4 weeks of blocking post-seizure neurogenesis suppresses chronic seizures. $\boldsymbol{A}$, Experimental design. $\boldsymbol{B}$, Graph showing frequency of SRS between Pilo/Veh/Veh and Pilo/GCV/GCV groups. Student's $t$ test, $p=0.0147$. C, Graph showing SRS duration between Pilo/Veh/Veh and Pilo/GCV/GCV groups. Student's $t$ test, $p=0.4351$. D, Graph showing time course analysis of seizure frequency of Pilo/Veh and Pilo/GCV groups over the period of $14 \mathrm{~d}$. E, Representative microscopic images showing DCXimmunoreactivity between Pilo/Veh/Veh and Pilo/GCV/GCV groups. Inset, Typical DCX+ cell. $\boldsymbol{F}$, Graph showing the number of DCX-expressing cells in the SGZ between the Pilo/Veh/Veh and Pilo/GCV/GCV groups. Student's $t$ test, $p=0.0055$. $\boldsymbol{G}$, Representative microscopic images showing Prox1 immunoreactivity of the mature granule neuron between Pilo/Veh/Veh and Pilo/GCV/GCV groups. Inset, Typical Prox1+ EGC. H, Graph showing Prox1+ EGCs in the Pilo/Veh/Veh and Pilo/GCV/GCV groups. Student'st test, $p=0.0025$. Scale bar, $100 \mu \mathrm{m}$; inset, $25 \mu \mathrm{m} . N=8-9$ animals per group. ns $=$ not significant. ${ }^{*} p<0.05,{ }^{* *} p \leq$ $0.01,{ }^{* * * *} p \leq 0.0001$.

total number of BrdU/Prox1+ ectopic cells in dentate hilus of Veh- and GCV-treated animals. We found that with 4 weeks of ablation of post-seizure neurogenesis, there were $584 \pm 107$ BrdU/Prox1+ cells in the hilus of GCV-treated animals compared with $1672 \pm 420.59$ cells in Veh-treated animals (Fig. $4 A, B)$. Next, we examined the total number of BrdU/Proxl+ cells in the dentate hilus after 8 weeks of blocking post-seizure neurogenesis (8-WPSE). We found that the number of BrdU/ Prox $1+$ cells was significantly reduced in GCV-treated (112.8 \pm 50.22) compared with Veh-treated (504 \pm 137.31 ) group of animals (Fig. $4 C, D, G, H$ ). To gain further insight into the long-term survival of the neural progenitors dividing at the time of acute seizures, we performed histology at 20-WPSE. The total number of BrdU/Prox1+ cells were still significantly reduced in GCVtreated animals $(38.4 \pm 5.879)$ compared with Veh-treated $(232.00 \pm 41.876)$ group of animals (Fig. 4E,F). Therefore, even though the number of hilar ectopic granule cells that originated from the cohort of neural progenitors dividing at the time of acute seizures is reduced both in the Veh- and GCV-treated group of animals at 8-WPSE and 20-WPSE, the seizure frequency is reduced only during continuous ablation for $>4$ weeks. This suggests that although continuous blocking of seizure-induced neurogenesis can control the development of seizures, other factors along with neurogenesis may play a role later in the progression of the disease.

\section{Discussion}

A characteristic feature of acquired epilepsy is the presence of a latent period followed by an initial brain insult. This latent period also described as the period of epileptogenesis is marked by extensive cellular and molecular changes that contribute to the development of chronic seizures (Pitkänen and Sutula, 2002). Nevertheless, it provides a clinically relevant time period where therapeutic interventions can be made to prevent the progression of epilepsy. Here, we show in a pilocarpine-induced SE model of mTLE in mice that continuous and concurrent blocking of postseizure neurogenesis for $>4$ weeks after acute seizures suppresses SRS frequency by $65 \%$. However, the suppression of seizures is transient and does not persist. These results suggest that (1) continuous reduction of a restricted post-injury cohort of new neurons can control the development of SRS transiently and (2) with the passage of time, the generation of additional cohorts of new 


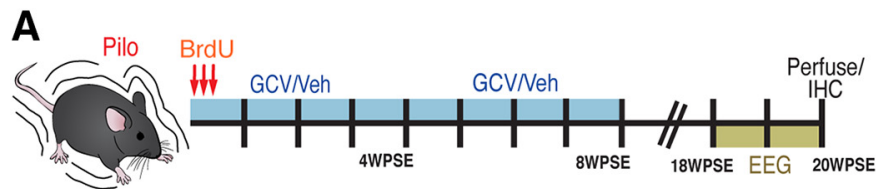

6 week old Nestin-TK
B

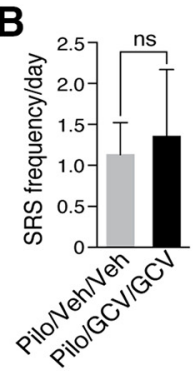

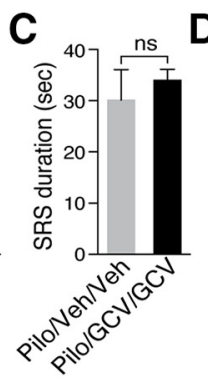

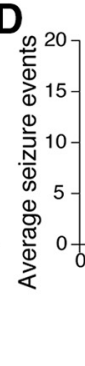

$\mathbf{E}$
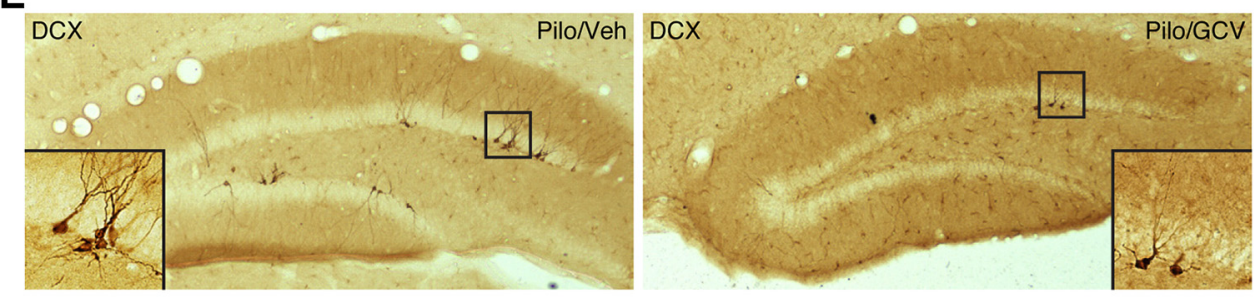

G
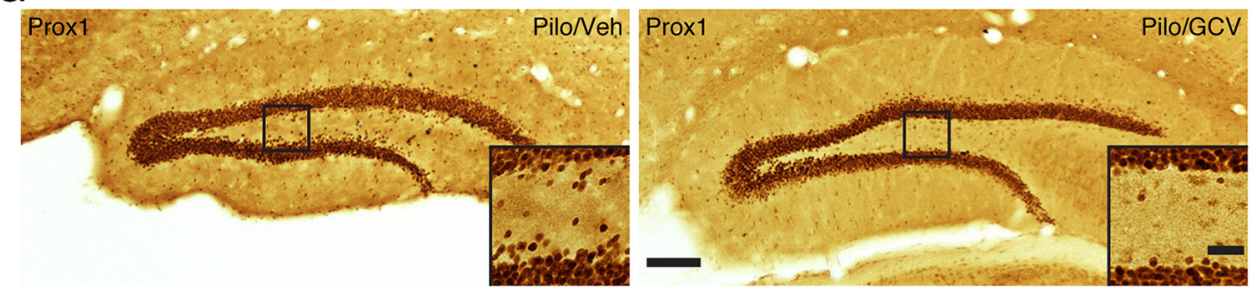

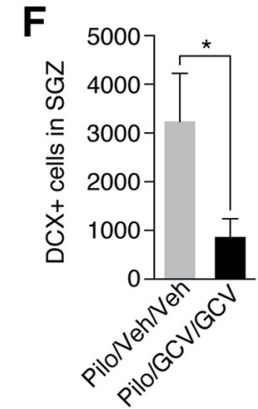

$\mathbf{H}$

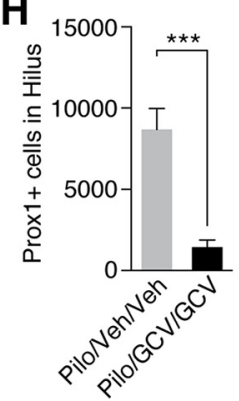

Figure 3. Reduced seizures from 8 weeks of post-seizure ablation does not persist. $A$, Experimental design. $\boldsymbol{B}$, Graph showing frequency of SRS between Pilo/Veh/Veh and Pilo/GCV/GCV groups. Student's $t$ test, $p=0.7819$. C, Graph showing SRS duration between Pilo/Veh/Veh and Pilo/GCV/GCV groups. Student's $t$ test, $p=0.5698$. $D$, Graph showing time course analysis of seizure frequency of Pilo/Veh and Pilo/GCV groups over the period of $14 \mathrm{~d}$. $\boldsymbol{E}$, Representative microscopic images showing DCX immunoreactivity between Pilo/Veh/Veh and Pilo/GCV/GCV groups. Inset, Typical DCX + cell. $F$, Graph showing the number of DCX-expressing cells in the SGZ between the Pilo/Veh/Veh and Pilo/GCV/GCV groups. Student's $t$ test, $p=0.0657$. G, Representative microscopic images showing Prox1 immunoreactivity of the mature granule neuron. Inset, Typical Prox1 + EGC. H, Graph showing Prox1 + EGCs in the Pilo/Veh/Veh and Pilo/GCV/GCV groups. Student's t test, $p=0.0004$. Scale bar, $100 \mu \mathrm{m}$; inset, $25 \mu \mathrm{m} . N=5-7$ animals per group. ns $=$ not significant. ${ }^{*} p<0.05,{ }^{* * *} p \leq 0.001$.

neurons along with extrahippocampal factors contributes to overall network hyperexcitability and epilepsy.

Despite decades of work, the very existence of human hippocampal neurogenesis is still a subject for debate. The first evidence of human hippocampal neurogenesis was shown 20 years ago and since then, there have been many studies either supporting or questioning the existence and relevance of human hippocampal neurogenesis (Eriksson et al., 1998; Spalding et al., 2013; Kempermann et al., 2018; Gage, 2019). A study published last year examined 18 adult and 19 perinatal or postnatal samples of postmortem brain tissue from individuals ranging from 14 gestational weeks to 77 years of age. They analyzed markers for proliferation, immature neurons, radial glia-like stem cells, and glia in the hippocampus and found that the highest number of immature neurons existed in the first years of life in the DG and dropped significantly with age. They did not detect any immature neurons in adult patients with epilepsy or heathy adults (Sorrells et al., 2018). By contrast, another study at the same time examined 28 postmortem hippocampi and found that in samples from healthy adults, hippocampal neurogenesis persisted until the eighth decade of life. However, they did observe a reduction in quiescent neural progenitor cells selectively in anterior-mid DG in aging individuals (Boldrini et al., 2018). Another recent study identified thousands of immature neurons in the DG of 13 neurologically healthy subjects between 43 and 87 years of age. Moreover, they also studied a cohort of 45 patients with Alzheimer's disease between 52 and 97 years of age and found that number of immature neurons declined progressively as the disease advanced (Moreno-Jiménez et al., 2019). All of these studies are aware of the limitations with postmortem brain samples and techniques of preservation as the methodological variances can lead to different conclusions about the existence of new neurons in the adult and aging brain. Nonetheless, these studies highlight the dynamic nature of adult hippocampal neurogenesis in humans under physiological and diseased conditions and also reignite the hope that manipulation of new neurons could someday help human patients.

Decades of work has shown that adult hippocampal neurogenesis in rodents is a tightly orchestrated cellular and molecular process. In this process, neural stem cells go through various developmental stages over a period of 4-6 weeks to become mature granule neurons that integrate into the existing dentate circuitry. These developmental stages include proliferation, differentiation, migration, axon/dendritic targeting, and synaptic integration (Hsieh, 2012). Whereas in normal brain, this stepwise process generates granule cells with usual functional properties, 
A

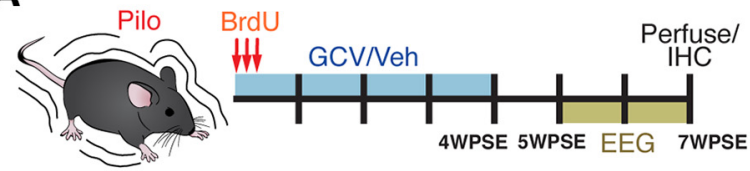

6 week old Nestin-TK

\section{C}

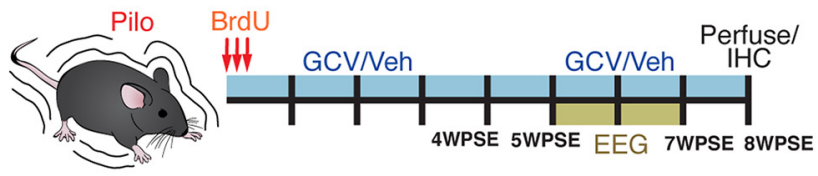

6 week old Nestin-TK

\section{E}

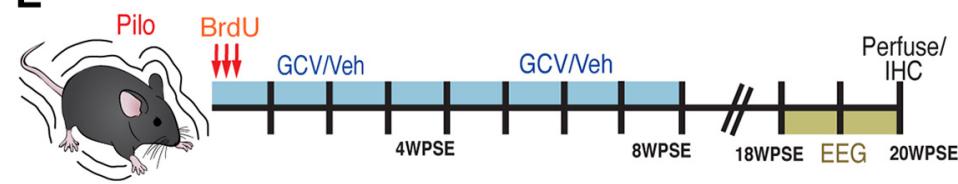

6 week old Nestin-TK

G
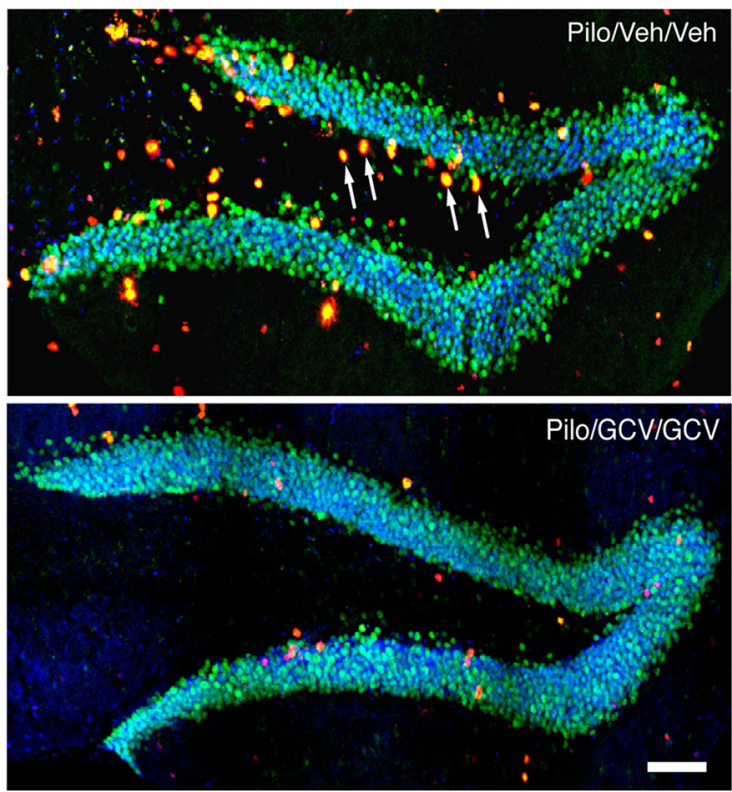

H
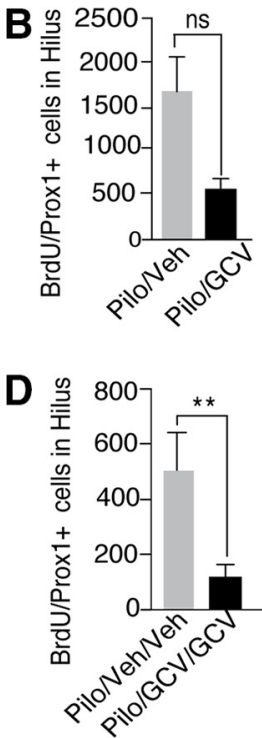

F

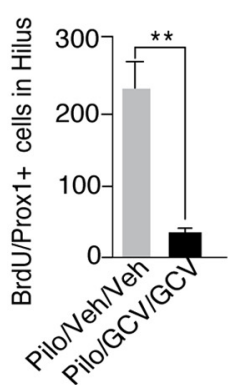

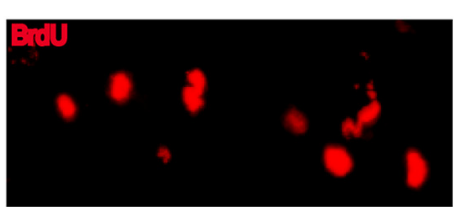
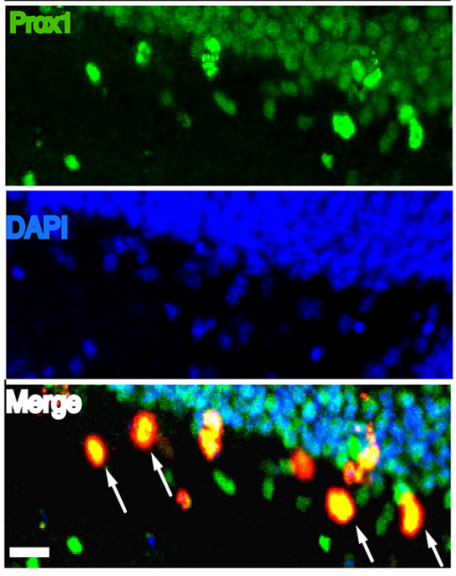

Figure 4. Effect of ablation of seizure-induced neurogenesis on survival of new neurons. $\boldsymbol{A}$, Experimental design for 4 weeks of ablation of post-seizure neurogenesis. $\boldsymbol{B}$, Graph showing the number of BrdU/Prox $1+$ cells between Pilo/Veh and Pilo/GCV groups. Student's $t$ test, $p=n$ s. C, Experimental design for 8 weeks of ablation of post-seizure neurogenesis. $\boldsymbol{D}$, Graph showing the number of BrdU/Prox1 + cells between Pilo/Veh/Veh and Pilo/GCV/GCV groups. Student's $t$ test, $p=0.0066$. $\boldsymbol{E}$, Experimental design for 8 weeks of ablation of post-seizure neurogenesis and recording at 20-WPSE. $F$, Graph showing the number of BrdU/Prox1+ cells between Pilo/Veh/Veh and Pilo/GCV/GCV groups. Student's $t$ test, $p=0.0025$. G, Representative microscopic images showing BrdU/Prox1 immunoreactivity of the ectopic granule neurons. Arrowheads show typical BrdU/Prox1 + EGCs. H, Magnified images of a typical BrdU/Prox1 + EGC. Scale bar, $100 \mu \mathrm{m}$; inset, $25 \mu \mathrm{m} . \mathrm{ns}=$ not significant. ${ }^{* *} p \leq 0.01$.

the scenario in an epileptic brain is different. The new neurons in an epileptic brain exhibit abnormal migration, develop hilar basal dendrites, contribute to mossy fiber sprouting, and show accelerated maturation and integration (Ribak et al., 2000; Schar- fman et al., 2000; Overstreet-Wadiche et al., 2006; Shapiro and Ribak, 2006; Shapiro et al., 2007, 2008; Walter et al., 2007). Retrovirus mediated labeling of different cohorts of new neurons born before and after seizures has shown that the impact on the 
A

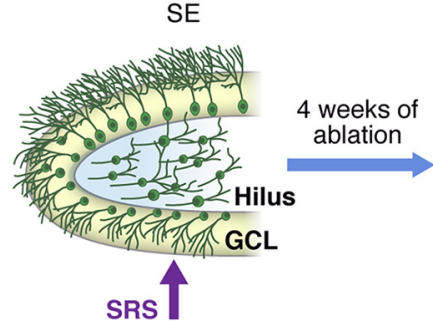

B

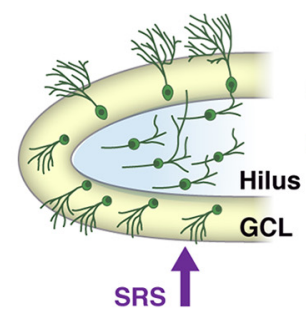

C

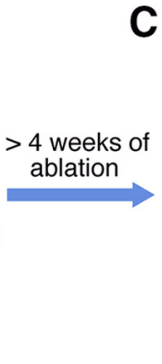

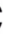

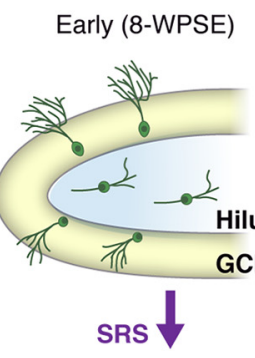

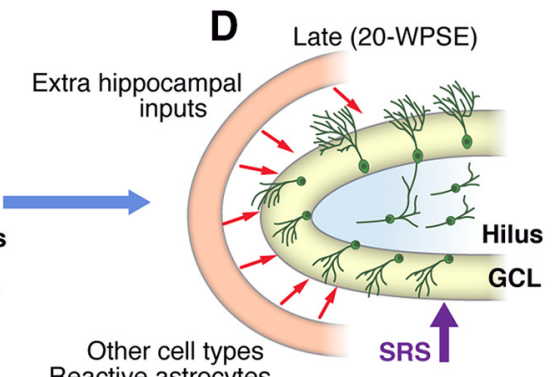

Reactive astrocytes

Reactive astrocytes
Mossy fiber sprouting

Figure 5. A model summarizing the observations in the study. $\boldsymbol{A}$, A pictorial representation of seizure-induced neurogenesis in an epileptic dentate gyrus. $\boldsymbol{B}$, Four weeks of ablation of post-seizure neurogenesis decreases neurogenesis but there is no change in seizure frequency. $\boldsymbol{C}$, Greater than 4 weeks of ablation further reduces seizure-induced neurogenesis and consequently reduces $S R S$ frequency. $\boldsymbol{D}$, The reduction in SRS with 8 weeks of ablation of post-seizure neurogenesis does not persist until 20-WPSE possibly because of resumed neurogenesis, extrahippocampal factors and/or the role of other cell types in the epileptic brain.

morphological and functional properties of new neurons and their contribution to the epileptogenic process is dependent on their time of birth in relation to epileptic insult (Jessberger et al., 2007). Moreover, studies have shown that not all new neurons have morphological and functional abnormalities and there is only a subset of newborn cohort of cells that are functionally disrupted suggesting the presence of functional heterogeneity among new neurons and their differential contribution to the epileptogenic process (Jakubs et al., 2006; Iyengar et al., 2015). For example, a detailed morphological characterization of agedefined cohort of new neurons has shown that neurons exposed to epileptogenic insult exhibit reduced dendritic spine numbers suggesting reduced excitatory input to these cells. Interestingly, in the same study a significant subset of new neurons was shown to have higher spine numbers possibly contributing to the epileptogenic circuitry (Murphy et al., 2011). Thus, these studies imply that the cohort of new neurons born before and after the epileptogenic insult are functionally heterogeneous; where some new neurons have protective or neutral roles and others display a disruptive role in the dentate circuitry. Previous work from our laboratory has shown that ablation of new neurons up to 4 weeks before pilocarpine-induced SE reduced seizure-induced neurogenesis by $98 \%$ and led to suppression of SRS frequency by $\sim 40 \%$ (Cho et al., 2015). Using a different transgenic mouse model to ablate adult neurogenesis (Hosford et al., 2016) showed that neurons born up to 5 weeks before SE can be ablated beginning $3 \mathrm{~d}$ after the epileptogenic insult and can lead to $50 \%$ reduction in seizures. Here, we show that 4 weeks of ablation of new neurons immediately after acute seizures did not have an effect on SRS frequency despite a significant reduction in the seizure-induced neurogenesis. We believe that this could be because of the following reason. Even after a substantial decrease in seizure-induced neurogenesis, there is still a population of remaining hilar EGCs $(\sim 2000)$ in GCV-treated group of animals. Although this number is reduced compared with $\sim 7500$ cells in Veh-treated animals, they may still function as hyperexcitable hub-like cells contributing to the epileptic circuit. Consistent with the idea, computational modeling and patch-clamp electrophysiology studies suggest hyperexcitable hub-like cells contribute to overall hippocampal network excitability and recurrent seizures (Scharfman et al., 2007; Morgan and Soltesz, 2008). Although 4 weeks of post-seizure ablation did not impact SRS, we show that continuous ablation of post-seizure neurogenesis for $>4$ weeks can suppress chronic SRS by 65\%. In GCV-treated animals after 8 weeks of post-seizure ablation, there were $\sim 680$ hilar EGCs compared with $\sim 2000$ cells after 4 weeks of ablation. Although these results are exciting because it suggests that continuous ablation of post-SE neurogenesis is required for seizure control, even a small number of residual hub-like EGCs may be sufficient to cause hyperexcitability because SRS were not completely absent after 8 weeks of ablation. We also show that even though the number of BrdU/ Prox $1+$ cells reduces significantly at both 8-WPSE and 20-WPSE, the SRS are impacted only during continuous blocking of seizure-induced neurogenesis. This again suggests that to effectively impact SRS, multiple cohorts of new neurons need to be blocked. Alternatively, this might also implicate that seizureinduced neurogenesis needs to be reduced below a certain threshold to reduce SRS. Complete ablation of neurogenesis after injury using pharmacological or genetic approaches could address the threshold idea. Moreover, because the new neurons are functionally heterogeneous, it is possible that ablation of post-seizure neurogenesis removes both the hyper and hypoexcitable seizureinduced new neurons. Therefore, in the future it would be essential to develop methodologies to tease apart the functionally heterogeneous new neurons and specifically target the aberrant new neurons below a critical threshold to impact SRS.

Because epilepsy is a lifelong debilitating disorder, any therapy for chronic seizures should have long-lasting effects. Therefore, we assessed whether the reduction of chronic SRS after 8 weeks of post-seizure ablation was sustained. Interestingly, despite a significant reduction in DCX + cells and Prox1 + hilar EGCs, there was no difference in the chronic SRS frequency between Veh- and GCV-treated groups of animals. These results are in contrast with our previous study by Cho et al. (2015), where ablation of neural progenitors before SE reduced seizure frequency up to 1 year. We believe that the different impact on SRS frequency between preand post-SE ablation could be because of several reasons. First, the study published by Cho et al. (2015) showed that pre-SE ablation of neurogenesis led to $>98 \%$ reduction of DCXexpressing cells. However, in the current study, the maximum ablation achieved was $\sim 89 \%$. Therefore, it is possible that once the ablation pumps are removed 8-WPSE, and with the passage of time, additional cohorts of new neurons born from the remaining cells alter the threshold to reduce SRS leading to overall network hyperexcitability and epilepsy. A second reason could be that this new cohort of cells is functionally more aberrant and contributes to SRS. Indeed, we observe the number of hilar EGCs was greater at 20 -WPSE $(\sim 1500$ cells $)$ compared with 8 -WPSE ( $\sim 680$ cells). This addition of new hyperexcitable EGCs can disrupt the functional integrity of the dentate circuit causing SRS. We also saw an increase in the number of DCX + cells from $\sim 656$ cells at 8 weeks post-SE to $\sim 921$ cells at 20 -WPSE suggesting that 
these new neurons could be contributing toward the network excitability. In future studies, it would be interesting to delay GCV treatment until 8-WPSE to test the contribution of additional cohorts of new neurons in SRS development.

Another interesting and important aspect of our study is that without GCV treatment, seizure frequency of the animals continues to increase up to 7-WPSE and decline significantly over the period of 20 weeks (Figs. 1D, 2D, 3D). This is partially in accordance with some studies on human and rodent models of mTLE, which suggest that seizure frequency in acquired epilepsy increases and then plateaus after several months (Bertram and Cornett, 1994; Cole, 2000). Additionally, it has also been shown in some studies that not all the animals display a progressive increase in seizure frequency, and some remain non-progressive (Gorter et al., 2001). We also observe that control animals in the second experimental paradigm have more seizures compared with the controls in the first experimental paradigm. We believe that this could be because of variability of seizure development among different cohorts of animals and the added stress of two rounds of pump implantation surgeries. Even in the same cohort of animals, we observe variability in seizure progression and frequency as evident from time course analysis (Figs. $1 D, 2 D, 3 D$ ). Studies suggest that environmental factors including stress can potentially exacerbate epileptic phenotype in humans (McKee and Privitera, 2017). Interestingly, we observe that with GCV treatment, the seizure frequency appears to be same in all three experimental paradigms. This observation strengthens our claim that targeting seizure-induced neurogenesis in a clinically relevant time period could potentially control the severity of the progression of disease even if not completely rescuing it. Though these results are exciting and informative, we are cautious regarding the interpretation and in the future, an ideal experiment would involve having the same animals being recorded multiple times over prolonged periods to fully elucidate the time course of mTLE in control and treated animals. Additionally, the role of diminished neurogenesis with aging also needs to be investigated to correctly interpret the changes in seizure frequency over time. For example, it has been shown that there is a dramatic decrease in neurogenesis and increase in seizure frequency in chronically epileptic mice $\sim 5$ months from SE (Hattiangady et al., 2004). However, it is not clear whether the decrease in neurogenesis is solely because of age or the chronically epileptic dentate gyrus contributes to the decline of neurogenesis and in turn increases seizure frequency. In future studies, age matched controls could help in understanding the combined role of chronically epileptic brain and diminished neurogenesis. It is also possible with the passage of time, extrahippocampal factors could contribute to overall network hyperexcitability and epilepsy. In fact, the pilocarpine model is known to be a nonspecific chemoconvulsant with broad effects in the entorhinal cortex, amygdala, and thalamus (Jung et al., 2009). Extrahippocampal inputs from these brain regions could mediate recurrent excitatory circuitry and lead to overall network excitability. To test the specific contribution of post-SE new neurons in a hippocampus-only epilepsy model, one may need to use alternative models such as the intrahippocampal kainate model of mTLE (Leite et al., 2002). Figure 5 summarizes the observations in this study. Finally, other cellular elements such as mossy fiber sprouting or reactive astrocytes post-SE may contribute to the long-term impact on SRS. Although this was not investigated in the current study, our previous study by Cho et al. (2015) showed ablation of reactive astrocytes correlated with the lack of seizure reduction consistent with the possible role of other cell types (Cho et al., 2015).
Epilepsy is a complex disease because of the numerous pathological alterations that co-occur during the epileptogenic period. Our study presented here not only supports the idea that it is possible to intervene therapeutically after the initial brain insult and temporarily improve pathophysiology but also cautions that targeting one specific cohort of new neurons might not be sufficient for long-term prevention of disease progression. A detailed cellular and molecular understanding of the latent period in terms of new neurons is critical for the development of antiepileptic drugs that are not only preventive but also curative.

\section{References}

Bertram EH, Cornett JF (1994) The evolution of a rat model of chronic spontaneous limbic seizures. Brain Res 661:157-162.

Boldrini M, Fulmore CA, Tartt AN, Simeon LR, Pavlova I, Poposka V, Rosoklija GB, Stankov A, Arango V, Dwork AJ, Hen R, Mann JJ (2018) Human hippocampal neurogenesis persists throughout aging. Cell Stem Cell 22:589-599.e5.

Brulet R, Zhu J, Aktar M, Hsieh J, Cho KO (2017) Mice with conditional NeuroD1 knockout display reduced aberrant hippocampal neurogenesis but no change in epileptic seizures. Exp Neurol 293:190-198.

Chang BS, Lowenstein DH (2003) Epilepsy. N Engl J Med 349:1257-1266.

Cho KO, Lybrand ZR, Ito N, Brulet R, Tafacory F, Zhang L, Good L, Ure K, Kernie SG, Birnbaum SG, Scharfman HE, Eisch AJ, Hsieh J (2015) Aberrant hippocampal neurogenesis contributes to epilepsy and associated cognitive decline. Nat Commun 6:6606.

Cole AJ (2000) Is epilepsy a progressive disease? The neurobiological consequences of epilepsy. Epilepsia 41:S13-S22.

D'Ambrosio R, Miller JW (2010) What is an epileptic seizure? unifying definitions in clinical practice and animal research to develop novel treatments. Epilepsy Curr 10:61-66.

Dudek FE, Bertram EH (2010) Counterpoint to "what is an epileptic seizure?” by D’Ambrosio and Miller. Epilepsy Curr 10:91-94.

Engel J Jr (2001) Mesial temporal lobe epilepsy: what have we learned? Neuroscientist 7:340-352.

Epp JR, Silva Mera R, Köhler S, Josselyn SA, Frankland PW (2016) Neurogenesis-mediated forgetting minimizes proactive interference. Nat Commun 7:10838.

Eriksson PS, Perfilieva E, Björk-Eriksson T, Alborn AM, Nordborg C, Peterson DA, Gage FH (1998) Neurogenesis in the adult human hippocampus. Nat Med 4:1313-1317.

Gage FH (2019) Adult neurogenesis in mammals. Science 364:827-828.

Gage FH, Temple S (2013) Neural stem cells: generating and regenerating the brain. Neuron 80:588-601.

Gorter JA, van Vliet EA, Aronica E, Lopes da Silva FH (2001) Progression of spontaneous seizures after status epilepticus is associated with mossy fibre sprouting and extensive bilateral loss of hilar parvalbumin and somatostatin-immunoreactive neurons. Eur J Neurosci 13:657-669.

Hattiangady B, Rao MS, Shetty AK (2004) Chronic temporal lobe epilepsy is associated with severely declined dentate neurogenesis in the adult hippocampus. Neurobiol Dis 17:473-490.

Hosford BE, Liska JP, Danzer SC (2016) Ablation of newly generated hippocampal granule cells has disease-modifying effects in epilepsy. J Neurosci 36:11013-11023.

Hosford BE, Rowley S, Liska JP, Danzer SC (2017) Ablation of peri-insult generated granule cells after epilepsy onset halts disease progression. Sci Rep 7:18015.

Hsieh J (2012) Orchestrating transcriptional control of adult neurogenesis. Genes Dev 26:1010-1021.

Iyengar SS, LaFrancois JJ, Friedman D, Drew LJ, Denny CA, Burghardt NS, Wu MV, Hsieh J, Hen R, Scharfman HE (2015) Suppression of adult neurogenesis increases the acute effects of kainic acid. Exp Neurol 264: $135-149$.

Jakubs K, Nanobashvili A, Bonde S, Ekdahl CT, Kokaia Z, Kokaia M, Lindvall O (2006) Environment matters: synaptic properties of neurons born in the epileptic adult brain develop to reduce excitability. Neuron 52:10471059.

Jessberger S, Römer B, Babu H, Kempermann G (2005) Seizures induce proliferation and dispersion of doublecortin-positive hippocampal progenitor cells. Exp Neurol 196:342-351.

Jessberger S, Zhao C, Toni N, Clemenson GD Jr, Li Y, Gage FH (2007) 
Seizure-associated, aberrant neurogenesis in adult rats characterized with retrovirus-mediated cell labeling. J Neurosci 27:9400-9407.

Jung KH, Chu K, Kim M, Jeong SW, Song YM, Lee ST, Kim JY, Lee SK, Roh JK (2004) Continuous cytosine-b-D-arabinofuranoside infusion reduces ectopic granule cells in adult rat hippocampus with attenuation of spontaneous recurrent seizures following pilocarpine-induced status epilepticus. Eur J Neurosci 19:3219-3226.

Jung KH, Chu K, Lee ST, Kim J, Sinn DI, Kim JM, Park DK, Lee JJ, Kim SU, Kim M, Lee SK, Roh JK (2006) Cyclooxygenase-2 inhibitor, celecoxib, inhibits the altered hippocampal neurogenesis with attenuation of spontaneous recurrent seizures following pilocarpine-induced status epilepticus. Neurobiol Dis 23:237-246.

Jung KH, Chu K, Lee ST, Kim JH, Kang KM, Song EC, Kim SJ, Park HK, Kim M, Lee SK, Roh JK (2009) Region-specific plasticity in the epileptic rat brain: a hippocampal and extrahippocampal analysis. Epilepsia 50: 537-549.

Kelly KM (2004) Spike-wave discharges: absence or not, a common finding in common laboratory rats. Epilepsy Curr 4:176-177.

Kempermann G, Wiskott L, Gage FH (2004) Functional significance of adult neurogenesis. Curr Opin Neurobiol 14:186-191.

Kempermann G, Gage FH, Aigner L, Song H, Curtis MA, Thuret S, Kuhn HG, Jessberger S, Frankland PW, Cameron HA, Gould E, Hen R, Abrous DN, Toni N, Schinder AF, Zhao X, Lucassen PJ, Frisén J (2018) Human adult neurogenesis: evidence and remaining questions. Cell Stem Cell 23: 25-30.

Kwan P, Brodie MJ (2000) Epilepsy after the first drug fails: substitution or add-on? Seizure 9:464-468.

Leite JP, Garcia-Cairasco N, Cavalheiro EA (2002) New insights from the use of pilocarpine and kainate models. Epilepsy Res 50:93-103.

Marucci G, Giulioni M, Rubboli G, Paradisi M, Fernández M, Del Vecchio G, Pozzati E (2013) Neurogenesis in temporal lobe epilepsy: relationship between histological findings and changes in dentate gyrus proliferative properties. Clin Neurol Neurosurg 115:187-191.

Mathern GW, Cifuentes F, Leite JP, Pretorius JK, Babb TL (1993) Hippocampal EEG excitability and chronic spontaneous seizures are associated with aberrant synaptic reorganization in the rat intrahippocampal kainate model. Electroencephalogr Clin Neurophysiol 87:326-339.

Mathern GW, Babb TL, Vickrey BG, Melendez M, Pretorius JK (1995) The clinical-pathogenic mechanisms of hippocampal neuron loss and surgical outcomes in temporal lobe epilepsy. Brain 118:105-118.

McKee HR, Privitera MD (2017) Stress as a seizure precipitant: identification, associated factors, and treatment options. Seizure 44:21-26.

Moreno-Jiménez EP, Flor-García M, Terreros-Roncal J, Rábano A, Cafini F, Pallas-Bazarra N, Ávila J, Llorens-Martín M (2019) Adult hippocampal neurogenesis is abundant in neurologically healthy subjects and drops sharply in patients with Alzheimer's disease. Nat Med 25:554-560.

Morgan RJ, Soltesz I (2008) Nonrandom connectivity of the epileptic dentate gyrus predicts a major role for neuronal hubs in seizures. Proc Natl Acad Sci U S A 105:6179-6184.

Murphy BL, Pun RY, Yin H, Faulkner CR, Loepke AW, Danzer SC (2011) Heterogeneous integration of adult-generated granule cells into the epileptic brain. J Neurosci 31:105-117.

Nadler JV (2003) The recurrent mossy fiber pathway of the epileptic brain. Neurochem Res 28:1649-1658.

Overstreet-Wadiche LS, Bromberg DA, Bensen AL, Westbrook GL (2006)
Seizures accelerate functional integration of adult-generated granule cells. J Neurosci 26:4095-4103.

Parent JM, Yu TW, Leibowitz RT, Geschwind DH, Sloviter RS, Lowenstein DH (1997) Dentate granule cell neurogenesis is increased by seizures and contributes to aberrant network reorganization in the adult rat hippocampus. J Neurosci 17:3727-3738

Pitkänen A, Sutula TP (2002) Is epilepsy a progressive disorder? prospects for new therapeutic approaches in temporal-lobe epilepsy. Lancet Neurol 1:173-181.

Racine RJ (1972) Modification of seizure activity by electrical stimulation: II. Motor seizure. Electroencephalogr Clin Neurophysiol 32:281-294.

Ribak CE, Tran PH, Spigelman I, Okazaki MM, Nadler JV (2000) Status epilepticus-induced hilar basal dendrites on rodent granule cells contribute to recurrent excitatory circuitry. J Comp Neurol 428:240-253.

Scharfman HE (2002) Epilepsy as an example of neural plasticity. Neuroscientist 8:154-173.

Scharfman HE, Goodman JH, Sollas AL (2000) Granule-like neurons at the hilar/CA3 border after status epilepticus and their synchrony with area CA3 pyramidal cells: functional implications of seizure-induced neurogenesis. J Neurosci 20:6144-6158.

Scharfman H, Goodman J, McCloskey D (2007) Ectopic granule cells of the rat dentate gyrus. Dev Neurosci 29:14-27.

Semah F, Picot MC, Adam C, Broglin D, Arzimanoglou A, Bazin B, Cavalcanti D, Baulac M (1998) Is the underlying cause of epilepsy a major prognostic factor for recurrence? Neurology 51:1256-1262.

Shapiro LA, Ribak CE (2006) Newly born dentate granule neurons after pilocarpine-induced epilepsy have hilar basal dendrites with immature synapses. Epilepsy Res 69:53-66.

Shapiro LA, Figueroa-Aragon S, Ribak CE (2007) Newly generated granule cells show rapid neuroplastic changes in the adult rat dentate gyrus during the first five days following pilocarpine-induced seizures. Eur J Neurosci 26:583-592.

Shapiro LA, Ribak CE, Jessberger S (2008) Structural changes for adultborn dentate granule cells after status epilepticus. Epilepsia 49:13-18.

Sorrells SF, Paredes MF, Cebrian-Silla A, Sandoval K, Qi D, Kelley KW, James D, Mayer S, Chang J, Auguste KI, Chang EF, Gutierrez AJ, Kriegstein AR, Mathern GW, Oldham MC, Huang EJ, Garcia-Verdugo JM, Yang Z, Alvarez-Buylla A (2018) Human hippocampal neurogenesis drops sharply in children to undetectable levels in adults. Nature 555:377-381.

Spalding KL, Bergmann O, Alkass K, Bernard S, Salehpour M, Huttner HB, Boström E, Westerlund I, Vial C, Buchholz BA, Possnert G, Mash DC, Druid H, Frisen J (2013) Dynamics of hippocampal neurogenesis in adult humans. Cell 153:1219-1227.

Thind KK, Ribak CE, Buckmaster PS (2008) Synaptic input to dentate granule cell basal dendrites in a rat model of temporal lobe epilepsy. J Comp Neurol 509:190-202.

von Campe G, Spencer DD, de Lanerolle NC (1997) Morphology of dentate granule cells in the human epileptogenic hippocampus. Hippocampus 7:472-488.

Walter C, Murphy BL, Pun RY, Spieles-Engemann AL, Danzer SC (2007) Pilocarpine-induced seizures cause selective time-dependent changes to adult-generated hippocampal dentate granule cells. J Neurosci 27:75417552.

Yu TS, Zhang G, Liebl DJ, Kernie SG (2008) Traumatic brain injuryinduced hippocampal neurogenesis requires activation of early nestinexpressing progenitors. J Neurosci 28:12901-12912. 